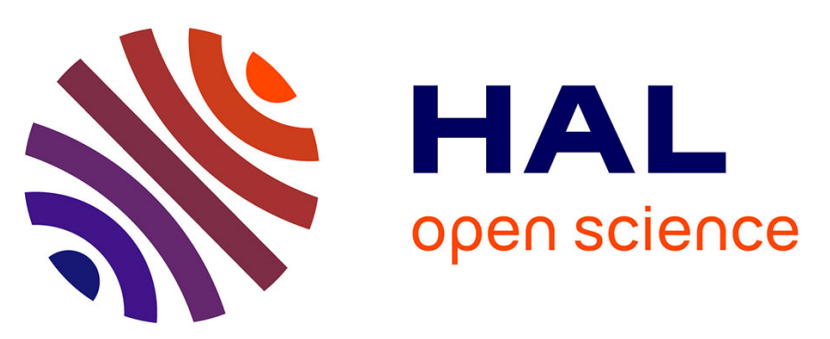

\title{
Apport de la diffraction de poudre à l'identification de composés minéraux dans quelques préparations pharmaceutiques
}

\author{
P. Renaud, Ch. Besselièvre, M. Broquaire
}

\section{- To cite this version:}

P. Renaud, Ch. Besselièvre, M. Broquaire. Apport de la diffraction de poudre à l'identification de composés minéraux dans quelques préparations pharmaceutiques. Journal de Physique IV Proceedings, 1996, 06 (C4), pp.C4-879-C4-885. 10.1051/jp4:1996484 • jpa-00254367

\section{HAL Id: jpa-00254367 https://hal.science/jpa-00254367}

Submitted on 1 Jan 1996

HAL is a multi-disciplinary open access archive for the deposit and dissemination of scientific research documents, whether they are published or not. The documents may come from teaching and research institutions in France or abroad, or from public or private research centers.
L'archive ouverte pluridisciplinaire HAL, est destinée au dépôt et à la diffusion de documents scientifiques de niveau recherche, publiés ou non, émanant des établissements d'enseignement et de recherche français ou étrangers, des laboratoires publics ou privés. 


\title{
Apport de la diffraction de poudre à l'identification de composés minéraux dans quelques préparations pharmaceutiques
}

\author{
P. Renaud, Ch. Besselièvre et M. Broquaire \\ Centre de Recherches du Laboratoire Louis Lafon, 19 avenue du Professeur Cadiot, 94700 Maisons-Alfort, \\ France
}

\begin{abstract}
Résumé : A partir de quelques exemples issus de leur laboratoire, les auteurs montrent l'intérêt que présente la diffraction de poudre appliquée à l'identification des composés minéraux entrant dans des préparations pharmaceutiques. Ils en discutent les avantages par rapport aux analyses chimiques traditionnelles proposées par les Pharmacopées.
\end{abstract}

Summary : Considering some examples coming from their laboratory, the autors demonstrate the interest of using powder diffraction for identifying mineral compounds in pharmaceuticals. They exhibit its advantages compared to classical chemical analyses proposed in Pharmacopoeia.

\section{INTRODUCTION}

Aujourd'hui, la diffraction de poudre reste une technique ignorée de la plus grande partie du monde pharmaceutique malgré la variété des problèmes qu'elle permet de résoudre [1]. Une telle ignorance se comprend mieux si l'on considère que la première proposition d'inclure la diffraction de poudre dans les techniques d'une pharmacopée européenne, date de Mars 1995 [2]. Elle figure à la Pharmacopée US mais il ne semble pas qu'il y soit fait appel dans les monographies.

Dans la seconde édition du "Handbook of Pharmaceutical Excipients [3]", parue en 1994, sur 204 monographies, 19 sont consacrées à des produits minéraux ; pour aucun d'entre eux, il n'est fait appel à une analyse de la forme cristalline par diffraction de poudre. La seule allusion au polymorphisme concerne l'oxyde de titane : l'anatase et le rutile sont caractérisés par leur densité (qui d'ailleurs se recouvrent), leur constante diélectrique et leur dureté. Aucune référence n'est faite à un quelconque diagramme de poudre.

Parmi les quelques 1060 monographies de la Pharmacopée Européenne [4] (document de référence, ayant force de loi sur l'ensemble des territoires de la C.E.E.) consacrées à des principes actifs, des excipients, des vaccins et divers produits à usage médical (fil pour sutures, coton hydrophile, etc.), 65 concernent des substances minérales et 748 des produits organiques ou des extraits végétaux. A aucun moment, il n'est fait référence à la diffraction de poudre comme technique d'identification de ces composés ; si cette situation peut se comprendre pour les produits organiques pour lesquels les analystes disposent de tout un arsenal de techniques très puissantes, il n'en va pas de même pour les produits minéraux : les méthodes 
d'identification font appel à des techniques purement chimiques, souvent très longues, peu automatisables et consommatrices de réactifs polluants de plus en plus coûteux à éliminer.

Ainsi l'identification du carbonate de calcium, choisi ici parce que c'est un excipient très classique et parce que les réactions d'identification des ions constituants figurent à la suite les unes des autres dans la Pharmacopée Européenne, demande trois analyses:

- les 2 réactions d'identité du calcium,

- la réaction des carbonates et bicarbonates,

mettant en oeuvre 10 réactifs différents [Fig. 1]. Un spectre de diffraction de poudre de carbonate de calcium de qualité suffisante pour son identification, peut être réalisé et comparé à un spectre de référence en moins de 20 minutes, temps considérablement plus court que celui nécessaire à la réalisation de l'identification chimique. De plus, le spectre de diffraction permet d'affirmer qu'il s'agit de carbonate de calcium pur, ce qui est impossible par la voie chimique, sauf à rajouter une recherche systématique d'ions.

\section{CALCIUM}

a) A $0,2 \mathrm{ml}$ d'une solution neutre contenant une quantité de la substance à examiner correspondant à $0,2 \mathrm{mg}$ environ de calcium $\left(\mathrm{Ca}^{2+}\right)$ par millilitre ou à $0,2 \mathrm{ml}$ de la solution prescrite, ajoutez $0,5 \mathrm{ml}$ d'une solution de glyoxalhydroxyanile $R$ à 0,2 pour cent $m / V$ dans l'alcool $\mathbf{R}, 0,2 \mathrm{ml}$ de solution diluée d'hydroxyde de sodium $\mathbf{R}$ et $0,2 \mathrm{ml}$ de solution de carbonate de sodium $\mathbf{R}$. Agitez avec $1 \mathrm{ml}$ à $2 \mathrm{ml}$ de chloroforme $\mathbf{R}$ et ajoutez $1 \mathrm{ml}$ à $2 \mathrm{ml}$ d'eau. La couche chloroformique est colorée en rouge.

b) Dissolvez $20 \mathrm{mg}$ environ de la substance à examiner ou la quantité prescrite dans $5 \mathrm{ml}$ d'acide acétique $\mathbf{R}$. Ajoutez $0,5 \mathrm{ml}$ de solution de ferrocyanure de potassium $\mathbf{R}$. La solution demeure limpide. Ajoutez $50 \mathrm{mg}$ environ de chlorure d'ammonium R. Il se forme un précipité cristallin blanc.

\section{CARBONATES ET BICARBONATES}

Dans un tube à essai, introduisez $0,1 \mathrm{~g}$ de la substance à examiner et mettez en suspension dans $2 \mathrm{ml}$ d'eau ou utilisez $2 \mathrm{ml}$ de la solution prescrite. Ajoutez $3 \mathrm{ml}$ d'acide acétique dilué $\mathbf{R}$ et fermez immédiatement le tube à l'aide d'un bouchon traversé par un tube en verre coudé 2 fois à angle droit. La solution ou la suspension devient effervescente et dégage un gaz incolore et inodore. Chauffez doucement et receuillez le gaz dans $5 \mathrm{ml}$ de solution d'hydroxyde de baryum $\mathbf{R}$. Il se forme un précipité blanc qui se dissout par addition d'acide chlorhydrique $\mathbf{R} 1$ en excès.

Figure 1: Identification du carbonate de calcium selon la Pharmacopée Européenne, $2^{\text {nd }}$ édition. (Extrait du chapître V.3 METHODES CHIMIQUES, V.3.1 REACTIONS D'IDENTITE, V.3.1.1. REACTIONS D'IDENTITE DES IONS ET DES GROUPES FONCTIONNELS. Dans le texte original, les réactifs employés n'apparaissent pas en caractères gras : il s'agit ici d'en faire ressortir le nombre).

Identification of calcium carbonate following the European Pharmacopoeia 
Par ailleurs, tous les produits minéraux recensés dans la Pharmacopée Européenne ont une ou plusieurs fiches, selon leur polymorphisme ou leur état d'hydratation dans les fichiers ICDD.

Ce constat nous a conduit à étudier avec un intérêt accru l'apport de la diffraction de poudre à l'analyse des produits minéraux à caractère pharmaceutique, principe actif ou excipient. Le processus usuel de la fabrication d'un médicament débute par l'identification et l'analyse de chacun des constituants en temps que matière première puis par l'identification et le dosage de ces constituants dans le produit fini, comprimé, gélule, ampoule, pommade, sirop, etc. Pour des raisons de commodité, nous nous sommes bornés à étudier les formes solides et le présent article regroupe quelques exemples simples d'identification de produits minéraux purs ou en mélange dans une matrice complexe organique et minérale.

\section{MATERIEL ET METHODE}

\subsection{Matériel}

Le diffractomètre de poudre utilisé pour les identifications est un diffractomètre de paillasse PHILIPS PW 1840, équipé d'un tube générateur de rayons $\mathrm{X}$ à foyer long et fin à anticathode de chrome (le choix du tube est déterminé par l'utilisation habituelle du spectromètre à l'analyse de produits organiques : on obtient ainsi une meilleure résolution) et piloté à l'aide du logiciel PC/APD version 3.5B ; le logiciel sert aussi au traitement et à la présentation des résultats.

\subsection{Programmes d'acquisition des spectres de poudre}

Un programme d'analyse rapide, balayant en moins de 20 minutes un domaine en $2 \theta$ allant de 5 à $120^{\circ}$ par pas de $0,02^{\circ}$ à la vitesse de $0,1^{\circ} / \mathrm{sec}$, permet pour chaque produit nouveau (étudié pour la première fois dans notre laboratoire) de connaître la zone en $2 \theta$ analytiquement intéressante. Un programme d'analyse plus lent, balayant par pas de $0,02^{\circ}$, à la vitesse de $0,01 \% \mathrm{sec}$, un domaine variable adapté au produit analysé, permet d'obtenir des diffractogrammes de qualité satisfaisante pour entreprendre une recherche sur une base de données ICDD ou sur une base plus restreinte interne au laboratoire.

\subsection{Produits étudiés}

Les exemples présentés ci-dessous concernent trois spécialités dont deux sont des présentations différentes des mêmes principes actifs.

L'Ulfon, présenté en sachet (Ulfon poudre orale, Sachets) ou sous forme de tablette lyophilisée (Ulfon-Lyoc, lyophilisat oral) est un protecteur de la muqueuse gastrique dont les principes actifs sont l'aldioxa, mélange défini d'allantoïne et d'hydroxyde d'aluminium, (900 mg), le carbonate de calcium (500 mg) et l'alcloxa $(100 \mathrm{mg})$. Ce dernier et les excipients nécessaires à la mise en forme galénique, en quantité beaucoup plus faible, ne sont peu ou pas cristallisés.

Le Mélange Magnésien est composé de différents sels de magnésium dont l'hydrogénophosphate, l'excipient principal étant le mannitol. 


\section{RESULTATS}

La figure 2 regroupe les diffractogrammes de l'Ulfon poudre orale, Sachets, de l'Ulfon-Lyoc, Lyophilisat Oral, et de leurs constituants principaux, affectés d'un coefficient lié à leurs proportions pour l'aldioxa et le carbonate de calcium (L'alcloxa et le poloxamère sont présentés sans atténuation : leur faible intensité de diffraction et leur faible teneur rend illisible une représentation à l'échelle).

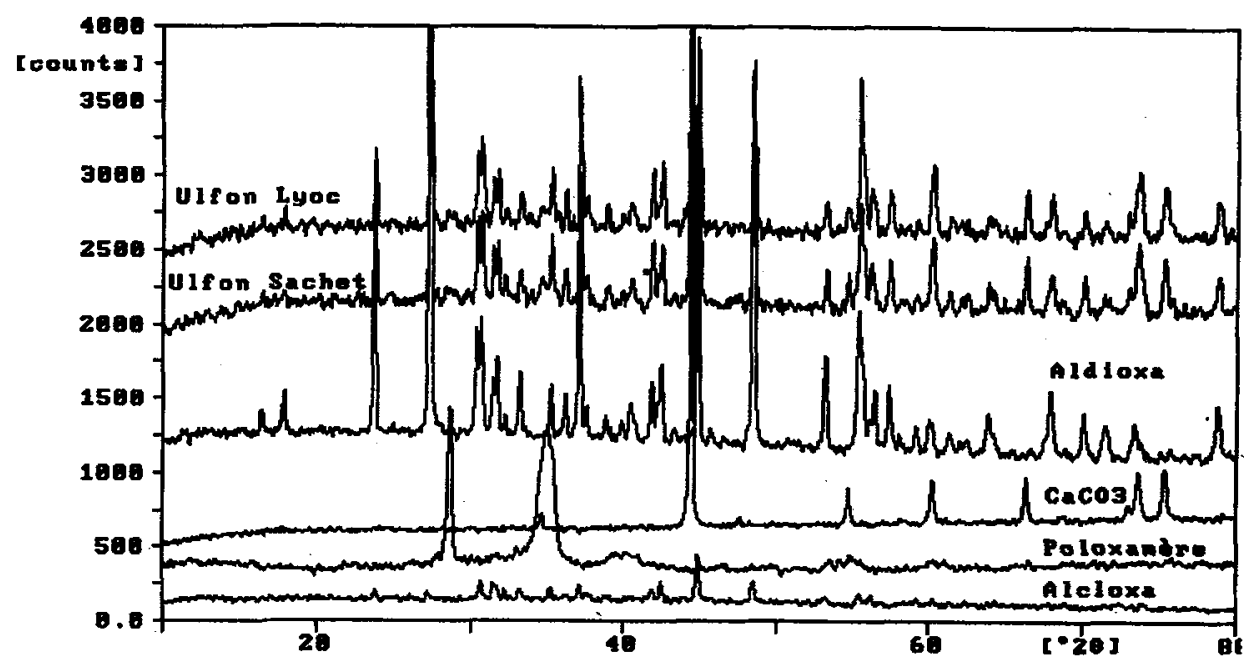

Figure 2: Diffractogrammes de l'Ulfon Sachets, de l'Ulfon-Lyoc et de leurs constituants

Diffractograms of Ulfon Sachets, Ulfon-Lyoc and their components.

L'analyse des diffractogrammes des constituants à l'état pur par recherche en bibliothèque et recherche de phase a permis d'identifier sans conteste le carbonate de calcium et, à partir du spectre de poudre de l'aldioxa, l'hydroxyde d'aluminium ; l'allantoïne n'a pu être identifiée à partir des fiches ICDD : comme beaucoup de produit organique, elle n'y figure pas ! Néanmoins, le spectre de diffraction de l'allantoïne et celui de l'aldioxa font maintenant partie de notre spectrothèque, ce qui en permet l'identification en routine par diffraction de poudre.

L'analyse des diffractogrammes des produits finis permet d'identifier sans difficulté la présence d'aldioxa et de carbonate de calcium ; les autres constituants sont en proportion trop faible pour pouvoir être identifiés. L'identification de l'aldioxa et du carbonate de calcium par les méthodes traditionnelles nécessite de réaliser :

- une réaction d'identification du calcium,

- une réaction d'identification de l'aluminium,

- une partie de la réaction d'identification des carbonates,

- et une CCM pour identifier l'allantoïne.

Par ailleurs, la comparaison des deux spectres Sachets et Lyoc montre qu'il n'y a pas de différence significative entre la poudre et le lyophilisat ; ce résultat est important : c'est un exemple de plus qui contredit le postulat selon lequel la lyophilisation rend amorphe un produit cristallisé. 
La figure 3 présente les diffractogrammes du Mélange Magnésien et de ses constituants principaux, affectés d'un coefficient tenant compte de leur proportion relative.

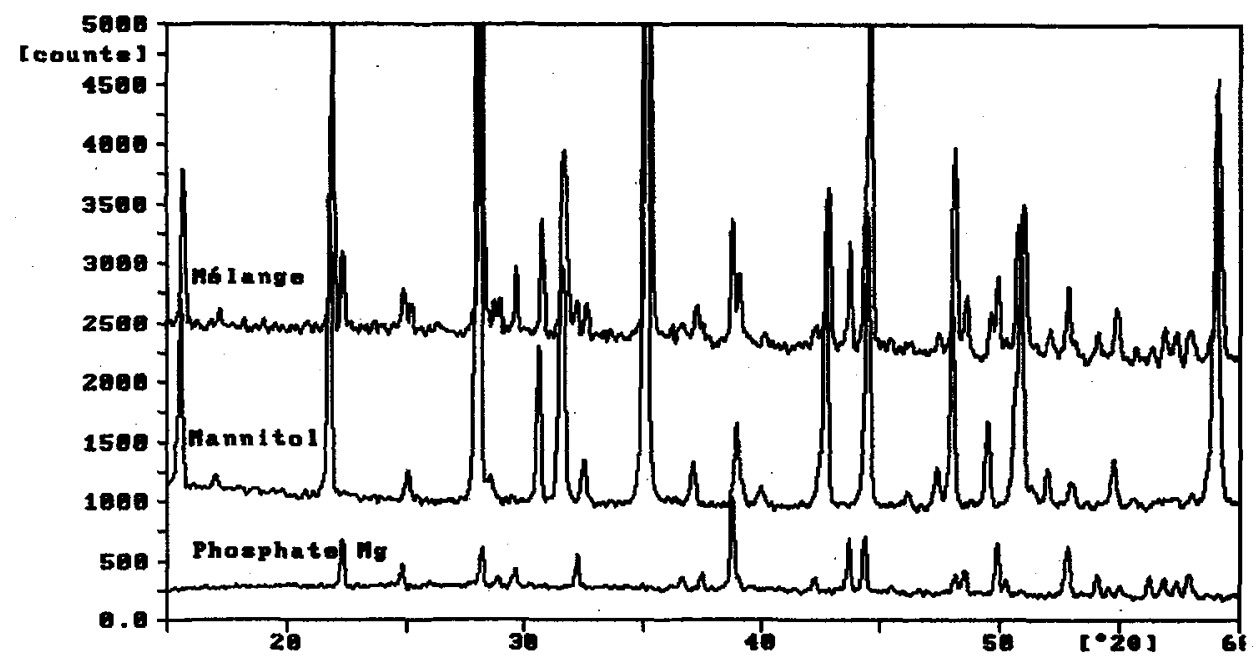

Figure 3: Diffractogramme du Mélange Magnésien et de ses principaux constituants

Diffractogram of Melange Magnesium and of its main components

L'analyse des spectres de poudre des constituants à l'état pur par recherche en bibliothèque et recherche de phase a permis de les identifier sans conteste et de manière bien plus simple qu'en suivant la Pharmacopée. Il est intéressant de noter que le diagramme qui fournit les pics de diffraction les plus intenses, est celui du mannitol, composé totalement organique, de formule brute $\mathrm{C}_{6} \mathrm{H}_{14} \mathrm{O}_{6}$. Cet exemple montre l'intérêt que peut présenter la diffraction de poudre en analyse organique.

L'analyse du diagramme du produit final permet l'identification simultanée du mannitol et du phosphate de magnésium. L'identification du phosphate de magnésium selon la Pharmacopée aurait nécessité :

- une calcination totale après attaque à l'acide sulfurique concentré (cendres sulfuriques), suivie de la réaction d'identification du magnésium,

- une mise en suspension du mélange, suivie d'une décantation puis de la réaction d'identification des phosphates appliquée à $1 \mathrm{ml}$ de surnageant.

Le mannitol est identifié par spectrométrie infrarouge du mélange poudre - bromure de potassium compacté.

\section{DISCUSSION}

Les exemples décrits ci-dessus, montrent l'intérêt que peut présenter la diffraction de poudre en laboratoire d'analyse et de contrôle pharmaceutique pour l'identification des produits minéraux avant ou après mise en forme galénique. Par rapport aux techniques d'identifications usuelles, telles que décrites dans la Pharmacopée, les avantages sont nombreux. 


\subsection{Spécificité de l'analyse}

Le spectre de diffraction d'un composé est une caractéristique physique de ce composé, liée comme son point de fusion à sa structure cristalline et à sa pureté. Deux produits différents ne peuvent avoir le même spectre de diffraction ; mais un même produit peut avoir plusieurs spectres de diffraction selon son polymorphisme ou ses états de solvatation : la diffraction de poudre permet donc l'identification, en une seule analyse, de ce produit sous une forme cristalline déterminée ou dans un état de solvatation donné. Une telle spécificité est impossible en utilisant uniquement les réactions d'identification des ions de la Pharmacopée: ainsi, il ne serait pas possible de différencier le dihydrogénophosphate de sodium, $\mathrm{NaH}_{2} \mathrm{PO}_{4}$, acidifiant usuel en Pharmacie Galénique, et le monohydrogénophosphate de sodium, $\mathrm{Na}_{2} \mathrm{HPO}_{4}$, alcalinisant.

\subsection{Facilité de l'analyse}

Pratiquement, un spectre de diffraction couvrant un domaine en $2 \theta$ compris entre 10 et $80^{\circ}$ obtenu en 10 à 15 minutes permet l'identification d'un composé minéral à partir des fiches ICDD ou des spectrothèques informatisées. Un seul programme d'analyse suffit donc pour l'identification de tous les produits minéraux susceptibles de rentrer dans une spécialité pharmaceutique ; la seule difficulté réside, comme pour tous les utilisateurs de diffraction de poudre, dans la préparation des échantillons soumis à l'analyse. Le traitement du résultat brut peut parfaitement être automatisé, l'analyse du diffractogramme, la recherche des pics et la comparaison en bibliothèque s'effectuant sans intervention extérieure. Dans ces conditions et en utilisant un passeur automatique, les opérations nécessaires à l'identification des produits analysés sont réduites et ne prennent que peu de temps à l'opérateur.

\subsection{Economie de l'analyse}

Au gain de temps obtenu par l'utilisation d'une machine surtout si elle est automatisée, s'ajoute un gain de temps important bien que difficile à évaluer : le temps consacré dans les analyses traditionnelles de la Pharmacopée à la gestion des nombreux réactifs nécessaires (achat, stockage, réapprovisionnement, préparation des solutions, durée de validité, procédure d'élimination des déchets, etc.). Par ailleurs, hormis l'investissement initial, le coût de fonctionnement d'un diffractomètre est faible et de toutes façons négligeable devant les coûts d'achat additionnés des réactifs d'identification et surtout devant les coûts de destruction après utilisation.

\subsection{Traçabilité de l'analyse}

Avec la généralisation des BPL (Bonnes Pratiques de Laboratoire) et des BPF (Bonnes Pratiques de Fabrication), l'identification des produits minéraux par diffraction de poudre a un avantage incontestable sur les techniques chimiques: la trace de l'analyse laissée par le spectre. Une fois l'analyse terminée, il reste une trace papier si on utilise un enregistreur, ou une trace informatique et papier si on utilise un logiciel d'acquisition et de traitement des données brutes. Etant donnée la tendance actuelle en matière de traçabilité, cet avantage de la diffraction de poudre sur les méthodes classiques de la Pharmacopée est certainement prépondérant. 


\section{CONCLUSION}

Les quelques résultats présentés ci-dessus ont permis de démontrer l'intérêt, tant technique qu'économique, de la diffraction de poudre pour l'identification rapide et univoque des composés minéraux entrant dans des préparations pharmaceutiques. Sur de nombreux points, le champ d'application de la diffraction des rayons $\mathrm{X}$ à l'identication des composés minéraux est comparable à celui de la spectrophotométrie infrarouge pour l'identification des produits organiques. Paradoxalement, l'examen même détaillé de ce travail apparemment novateur, montre qu'aucun résultat nouveau n'a été fourni, tout existait déjà et était parfaitement décrit : les spectres, leurs conditions d'obtention, etc., mais personne, aujourd'hui, en laboratoire de contrôle pharmaceutique n'utilise ces données. Peut-être, y a-t-il là un nouveau débouché pour la diffraction de poudre?

\section{REFERENCES}

[1] M. Broquaire et S. Raud, STP Pharma Pratiques 5 (4) 310-316 1995

[2] Pharmeuropa, Vol. 7,1, 52-53, 1995.

[3] Handbook of Pharmaceutical Excipients, 2nd Ed, A. Wade and P.J. Weller editors, Pharmaceutical Press, London, 1994.

[4] Pharmacopée Européenne, 2ème Edition, Maisonneuve Ed, Ste Ruffine, 1995 